\title{
Progress in the care of familial hypercholesterolaemia: 2016
}

\author{
Damon A Bell, ${ }^{1,2}$, Gerald F Watts ${ }^{1,2}$
}

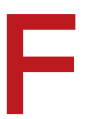

amilial hypercholesterolaemia $(\mathrm{FH})$ is the most common autosomal dominant condition. ${ }^{1} \mathrm{FH}$ reduces the catabolism of low-density lipoprotein cholesterol (LDL-c) and increases rates of premature atherosclerotic cardiovascular disease (CVD). This review focuses on recent advances in the management of $\mathrm{FH}$, and the implications for both primary and secondary care, noting that the majority of individuals with $\mathrm{FH}$ remain undiagnosed. ${ }^{2}$

$\mathrm{FH}$ was previously considered to have a prevalence of one in 500 in the general community, including in Australia. ${ }^{3}$ Recent evidence, however, suggests the prevalence is between one in 200 and one in 350 , which equates to over 30 million people estimated to have $\mathrm{FH}$ worldwide. ${ }^{4,5}$ These prevalence figures relate to the general population, and while $\mathrm{FH}$ is present in all ethnic groups, communities with gene founder effects and high rates of consanguinity, such as the Afrikaans, Christian Lebanese and Québécois populations, have a higher prevalence of the condition.

Further, the prevalence of homozygous or compound heterozygous $\mathrm{FH}$ has been demonstrated to be at least three times more common than previously reported, with a prevalence of about one in 300000 people in the Netherlands. ${ }^{4}$ The detection and management of individuals with homozygous $\mathrm{FH}$ has been described in a consensus report from the European Atherosclerosis Society. ${ }^{6}$ Homozygous FH is a very severe disorder, with untreated people often developing severe atherosclerotic CVD before 20 years of age. Such individuals often have LDL-c concentrations $>13 \mathrm{mmol} / \mathrm{L}$ and severe cutaneous and tendon xanthomata. While diet and statins are the mainstays of therapy, early intervention (before 8 years of age) with LDL apheresis or novel lipid-lowering medication, such as proprotein convertase subtilisin/kexin type 9 (PCSK9) inhibitors or microsomal triglyceride transfer protein inhibitors, is indicated. Patients with suspected homozygous FH should be referred to a specialist centre. ${ }^{6}$

Several recent international guidelines on the care of FH have been published. ${ }^{3,7-10}$ These have focused on early detection and treatment of individuals with FH. However, there is still no international consensus on the diagnostic criteria for $\mathrm{FH}$, or on the utility of genetic testing. The Dutch Lipid Clinic Network criteria (DLCNC) are preferred for diagnosing FH in index cases in Australia (Box 1$){ }^{2}$ The International FH Foundation guidance acknowledges geographical differences in care, and recognises the need for countries to individualise service delivery. CVD risk in $\mathrm{FH}$ is dependent on classic CVD risk factors. However, $\mathrm{FH}$ is appropriately excluded from general absolute CVD algorithms, since these underestimate the absolute risk in $\mathrm{FH}$.

FH guidelines provide therapeutic goals, which vary depending on the specific absolute CVD risk for patients with FH. In adults, the general LDL-c goal is a least a $50 \%$ reduction in pre-therapy LDL-c levels, followed by a target of LDL-c $<2.5 \mathrm{mmol} / \mathrm{L}$, or $<1.8 \mathrm{mmol} / \mathrm{L}$ in individuals with CVD or other major CVD risk factors; these international targets update those of previously published

\section{Summary}

- Familial hypercholesterolaemia (FH) is the most common autosomal dominant condition, with a prevalence of between one in 200 and one in 350 people in the general population.

- Untreated $\mathrm{FH}$ is associated with premature atherosclerotic cardiovascular disease (CVD).

- The prevalence of homozygous or compound heterozygous FH is now considered to be about one in 300000 people.

- Treating children with FH reduces progression of atherosclerotic CVD and future CVD events.

- Most individuals with FH are undiagnosed, which together with the recent frequency data in the population and in individuals with premature coronary disease creates a public health challenge and mandates a key role for primary care.

- Childhood is the optimal period for detecting FH, since lowdensity lipoprotein cholesterol (LDL-c) concentrations better differentiate affected from unaffected individuals.

- In an Australian community setting, over $70 \%$ of adults with an LDL-c level $\geq 6.5 \mathrm{mmol} / \mathrm{L}$ have clinical $\mathrm{FH}$; of these, $30 \%$ have a detectable mutation.

- The community laboratory has an important role in identifying $\mathrm{FH}$, with interpretive comments leading to additional reductions in LDL-c concentrations, and a phone call from the pathologist to the general practitioner improving detection of cases.

- Cascade screening using DNA testing is cost-effective and acceptable to screenees.

- Next generation genetic sequencing may differentiate people with polygenic hypercholesterolaemia alone from those with $\mathrm{FH}$.

- Smoking, hypertension, elevated lipoprotein(a) levels, chronic kidney disease and diabetes are additional atherosclerotic CVD risk factors in $\mathrm{FH}$.

- Equations for assessing absolute risk of CVD in primary prevention underestimate risk in $\mathrm{FH}$.

- The adult LDL-c goal is a greater than $50 \%$ reduction in LDL-c levels, followed by a target of $<2.5 \mathrm{mmol} / \mathrm{L}$, or $<1.8 \mathrm{mmol} / \mathrm{L}$ for individuals with CVD or other CVD risk factors.

- Proprotein convertase subtilisin/kexin type 9 inhibitors significantly reduce LDL-c and lipoprotein(a) levels in people with $\mathrm{FH}$.

- Registries are essential for improving the care of people with $\mathrm{FH}$.

Australian FH recommendations. ${ }^{2,3,10}$ Currently only about $20 \%$ of individuals with FH attain an LDL-c level $<2.5 \mathrm{mmol} / \mathrm{L}^{11}$

\section{Detecting $\mathrm{FH}$ in children}

The European Atherosclerosis Society published a guideline focusing on paediatric aspects of the diagnosis and treatment of children with FH in 2015.8 This guideline outlined the benefit of early treatment of children with FH using statins. There is a 
1 Dutch Lipid Clinic Network Criteria score for the diagnosis of familial hypercholesterolaemia $(\mathrm{FH})^{2}$

\section{Criteria}

Family history

First-degree relative with known premature coronary and/or vascular disease (men aged < 55

years, women aged $<60$ years); or

First-degree relative with known LDL-c > 95th percentile for age and sex

First-degree relative with tendon xanthomas and/ or arcus cornealis; or

Children aged $<18$ years with LDL-c $>95$ th

percentile for age and sex

Clinical history

Patient with premature coronary artery disease (ages as above)

Patient with premature cerebral or peripheral vascular disease (ages as above)

Physical examination

Tendon xanthomata

Arcus cornealis at age $<45$ years

LDL-C

$$
\begin{aligned}
& \geq 8.5 \mathrm{mmol} / \mathrm{L} \\
& 6.5-8.4 \mathrm{mmol} / \mathrm{L} \\
& 5.0-6.4 \mathrm{mmol} / \mathrm{L} \\
& 4.0-4.9 \mathrm{mmol} / \mathrm{L}
\end{aligned}
$$

DNA analysis: functional mutation in the LDL receptor, apolipoprotein B or PCSK9 gene

\section{Stratification}

Definite $\mathrm{FH}$

Probable $\mathrm{FH}$

Possible FH

$\mathrm{LDL}-\mathrm{C}=$ low-density lipoprotein cholesterol. PCSK9 = proprotein convertase subtilisin/kexin type 9 . significant difference in the carotid intima medial thickness (a measure of subclinical atherosclerosis) in children with $\mathrm{FH}$ and their unaffected siblings by 7 years of age, with implications for the value of early treatment. Lifestyle modifications and statins from 8 years of age can reduce the progression of atherosclerosis to the same rate as unaffected siblings over a 10 -year period. ${ }^{8}$ Early treatment of children improves CVD-free survival by 30 years of age $(100 \%)$ compared with their untreated parents $(93 \%$; $P=0.002) .{ }^{8}$ While further long term data on statin use in children are required, there are 10-year follow up data for children who were initiated on pravastatin between the ages of 8 and 18 years, which demonstrate that statin therapy is safe and effective. ${ }^{12}$ Hence, the balance of risk and benefit suggests that use of statins in children with FH is safe and efficacious, at least in the short to intermediate term, with all recommendations appropriately requiring that potential toxicity and adverse events be closely monitored.

Childhood is the optimal period for detecting FH, as LDL-c concentration is a better discriminator between affected and unaffected individuals in this age group. After excluding secondary causes and optimising lifestyle and repeating fasting
2 Protocol for genetic cascade screening in Western Australia*

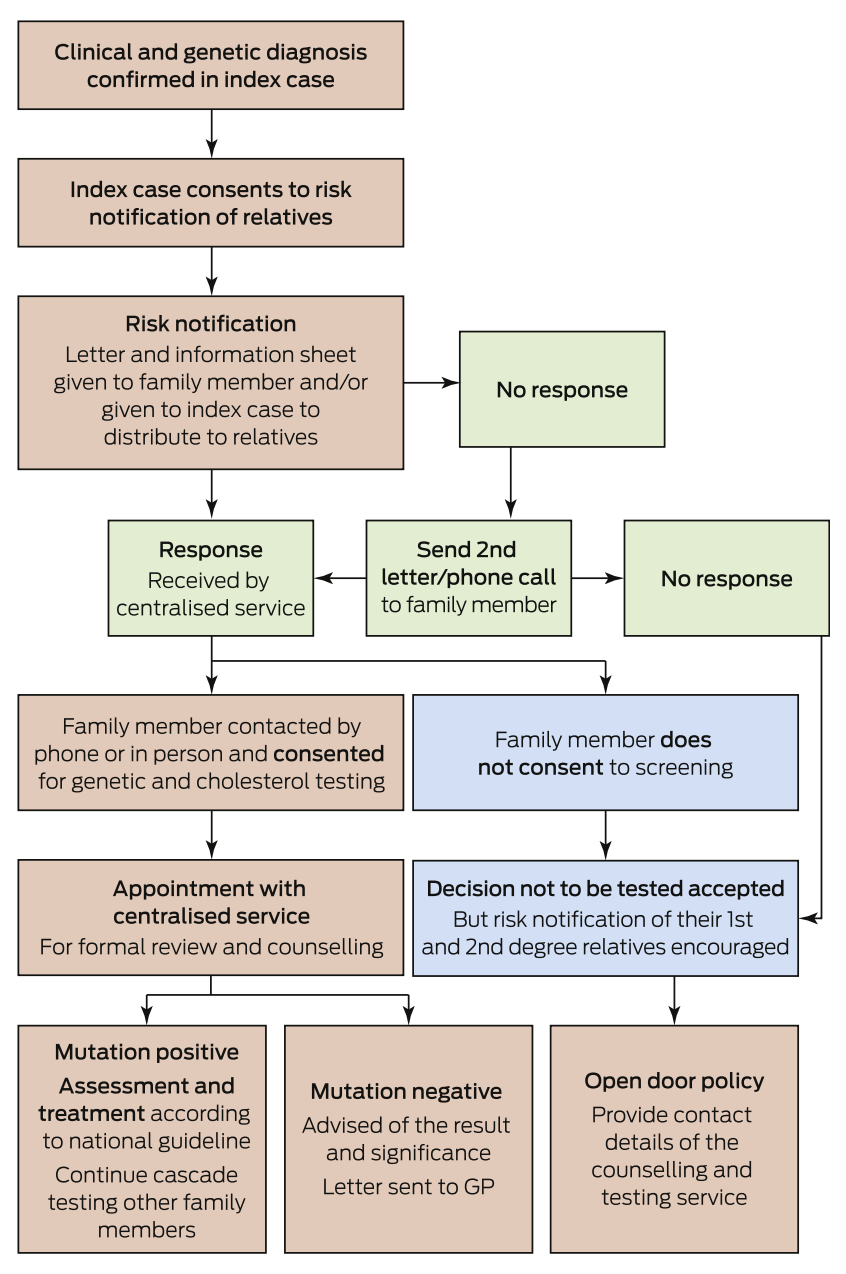

* Family cascade screening process performed according to national guidelines after obtaining written consent from the index case. "This was undertaken by a trained nurse who contacted the family members and obtained verbal consent to contact further family members, after providing counselling and offering specialist review as indicated.
LDL-c on two occasions, a child is considered likely to have FH if they have:

- $\quad$ an LDL-c level $\geq 5.0 \mathrm{mmol} / \mathrm{L}$;

- a family history of premature CVD and an LDL-C level $\geq 4.0 \mathrm{mmol} / \mathrm{L}$; or

- a first-degree relative with genetically confirmed FH and an LDL-c level $\geq 3.5 \mathrm{mmol} / \mathrm{L}$.

Universal screening for $\mathrm{FH}$ in children has been demonstrated to be effective in Slovenia, but experience is limited elsewhere. ${ }^{13}$ The therapeutic targets for children are less aggressive than for adults: a reduction in LDL-c of over 50\% in children aged 8-10 years and an LDL-c level $<3.5 \mathrm{mmol} / \mathrm{L}$ from the age of 10 years.

\section{Cascade screening}

Recent reports from Western Australia confirm that cascade screening is efficacious and cost-effective. ${ }^{11,14}$ The genetic cascade screening and risk notification process followed in Western Australia is shown in Box $2 .{ }^{11}$ Cascade screening involves testing close relatives of individuals diagnosed with $\mathrm{FH}$. The autosomal 
dominant inheritance suggests $50 \%$ of first-degree relatives would be expected to have $\mathrm{FH}$. Two new cases of $\mathrm{FH}$ were found by cascade screening for each index case in WA. ${ }^{11}$ These individuals were younger and had less atherosclerotic CVD than index cases. Interestingly, about $50 \%$ were already on lipid-lowering therapy, but they were not treated to the recommended goals and further lipid reductions were achieved overall. Over $90 \%$ of patients were satisfied with the cascade screening process and care provided by this service ${ }^{11}$ However, for individuals who have not had genetic testing performed, or for individuals with clinical $\mathrm{FH}$ in whom a mutation has not been identified, cascade screening should also be undertaken using LDL-c alone.

Although we have recently shown that genetic testing is costeffective in the cascade screening setting ( $\$ 4155$ per life year saved) ${ }^{14}$ only a few centres in Australia have this facility and testing is currently not Medicare rebatable. However, combining increased awareness of the benefits of identifying people with $\mathrm{FH}$ with the reducing analytical costs may increase the use of genetic testing. This in turn could guide advocacy and lobbying Medicare to support genetic testing for $\mathrm{FH}$.

\section{Detecting $\mathrm{FH}$ in the community}

A novel approach that utilises the community laboratory to augment the detection of FH has recently been tested in Australia. The community laboratory is well placed to perform opportunistic screening, since they perform large numbers of lipid profiles, the majority (>90\%) of which are requested by general practitioners. ${ }^{15}$ Clinical biochemists can append an interpretive comment to the lipid profile reports of individuals at high risk of $\mathrm{FH}$ based on their LDL-c results. These interpretive comments on high risk individuals (LDL-c $\geq 6.5 \mathrm{mmol} / \mathrm{L}$ ) led to a significant additional reduction in LDL-c and increased referral to a specialist clinic. ${ }^{16} \mathrm{~A}$ phone call from the clinical biochemist to the requesting GP improved both the referral rate of high risk (LDL-c $\geq 6.5 \mathrm{mmol} / \mathrm{L}$ ) individuals to a lipid specialist and the subsequent confirmation of phenotypic $\mathrm{FH}$ in $70 \%$ of those referred, with genetic testing identifying a mutation in $30 \%$ of individuals. ${ }^{17}$ There is a list of specialists with an interest in lipids on the Australian Atherosclerosis Society website (http://www.athero.org.au/fh/healthprofessionals/fh-specialists).

In Australia, GPs consider they are the best placed health professionals to detect and treat individuals with $\mathrm{FH}$ in the community. ${ }^{18}$ A large primary care $\mathrm{FH}$ detection program in a rural community demonstrated that using pathology and GP practice databases was the most successful method to systematically detect people with $\mathrm{FH}$ in the community. ${ }^{19}$ However, a survey of GPs uncovered some key knowledge deficits in the prevalence, inheritance and clinical features of $\mathrm{FH}$, which would need to be addressed before GPs can effectively detect and treat individuals with $\mathrm{FH}$ in the community. ${ }^{18}$ A primary care-centred FH model of care for Australia has recently been proposed to assist GPs with FH detection and management, but this requires validation. ${ }^{20}$ The model of care includes an algorithm that is initiated when an individual is found to have an LDL-c level $\geq 5.0 \mathrm{mmol} / \mathrm{L}$, which could be highlighted as at risk of FH by either a laboratory or the GP practice software. $^{20,21}$ The doctor is then directed to calculate the likelihood of FH using the DLCNC. Patients found to have probable or definite $\mathrm{FH}$ are assessed for clinical complexity and considered for cascade testing. See the Appendix at mja.com.au for the algorithm and definition of complexity categories. FH-possible patients should be treated according to general cardiovascular disease prevention guidelines.

\section{Molecular aspects}

There have also been advances in molecular aspects of $\mathrm{FH}$. A recent community-based study in the United States confirmed that among patients with hypercholesterolaemia, the presence of a mutation was independently predictive of CVD, underscoring the value of genetic testing. ${ }^{22}$ The mutation spectrum of $\mathrm{FH}$ was described in an Australian population and was found to be similar to that in Europe and the United Kingdom. ${ }^{23}$ Mutation detection yields in Australia are comparable with the international literature; for example, $70 \%$ of individuals identified with clinically definite FH (DLCNC score > 8) had an identifiable mutation, whereas only $30 \%$ of those with clinically probable $\mathrm{FH}$ had a mutation. ${ }^{23}$

Polygenic hypercholesterolaemia (multiple genetic variants that each cause a small increase in LDL-c but collectively have a major effect in elevating LDL-c levels) is one explanation for not identifying an FH mutation. An LDL-c gene score has been described to differentiate individuals with FH (lower score) from those with polygenic hypercholesterolaemia (higher score), but this requires validation. ${ }^{24}$ About $30 \%$ of individuals with clinical FH are likely to have polygenic hypercholesterolaemia, and cascade screening their family members may not be justified. ${ }^{25}$

A further possible explanation for failure to detect a mutation causative of FH in an individual with clinically definite FH may lie in the limitations of current analytical methods such as restricting analysis to panels of known mutations. Further, FH is genetically heterogeneous and there may be unknown alleles and loci that cause FH. Next generation sequencing is capable of sequencing the whole genome or targeted exomes rapidly at a relatively low cost, and may improve mutation detection and identify novel genes causing $\mathrm{FH}$, but further experience with its precise value in a clinical setting is required. Whole exome sequencing was able to identify a mutation causing $\mathrm{FH}$ in $20 \%$ of a cohort of "mutation negative" but clinically definite FH patients. ${ }^{25}$ However, when applied to patients with hypercholesterolaemia in a primary care setting, pathogenic mutations were only detected in $2 \%$ of individuals, with uncertain or non-pathogenic variants detected in a further $1.4 \% .^{26}$

\section{Cardiovascular risk assessment}

Absolute CVD risk assessment, employing risk factor counting, should be performed as atherosclerotic CVD risk is variable in FH. ${ }^{10,27}$ This involves appraisal of classic CVD risk factors including, age, sex, hypertension, diabetes, chronic kidney disease and smoking. The prevalence of classic CVD risk factors among Western Australians with recently identified $\mathrm{FH}$ was $13 \%$ for hypertension, $3 \%$ for diabetes and $16 \%$ for smokers, all of which were amenable to clinical intervention. ${ }^{11}$

Other non-classic CVD risk factors are also important for individuals with $\mathrm{FH}$, especially chronic kidney disease and elevated levels of lipoprotein(a). ${ }^{28}$ Lipoprotein(a) is a circulating lipoprotein consisting of an LDL particle with a covalently linked apolipoprotein A. Its plasma concentration is genetically determined and it is a causal risk factor for CVD in both the general population and FH patients. ${ }^{29,30}$ Lipoprotein(a) concentrations are not affected by diet or lowered by statins. ${ }^{31}$

\section{Management and new therapies}

The past 2 years have also seen the development of new treatments for $\mathrm{FH}$, but lifestyle modifications and statins remain the cornerstones of therapy for FH. Ezetimibe has been demonstrated to reduce coronary events against a background of simvastatin in 
non-FH patients with established CVD. ${ }^{32}$ PCSK9 inhibitors have recently been approved to treat individuals with $\mathrm{FH}$ or atherosclerotic CVD not meeting current LDL-c targets in Europe and America. PCSK9 is a hepatic convertase that controls the degradation and hence the lifespan of the LDL receptor. PSCK9 is secreted by the hepatocyte and binds to the LDL receptor on the surface of the hepatocyte. The LDL receptor-PCSK9-LDL-c complex is then internalised via clathrin-dependent endocytosis, but the PSCK9 directs the LDL receptor towards lysosomal degradation instead of recycling it back to the hepatocyte surface. ${ }^{33}$ A recent meta-analysis of early PCSK9 inhibition trials involving over 10000 patients demonstrated a 50\% reduction in LDL-c, a $25 \%$ reduction in lipoprotein(a), and significant reductions in allcause and cardiovascular mortality. ${ }^{34}$

The PCSK9 inhibitors alirocumab and evolocumab were approved by the European Medicines Agency in 2016 for homozygous and heterozygous $\mathrm{FH}$ and non-FH individuals unable to reach LDL-c targets, and for individuals with hypercholesterolemia who are statin intolerant. In the US, the Food and Drug Administration has approved alirocumab for heterozygous $\mathrm{FH}$ and individuals with atherosclerotic CVD who require additional reduction of LDL-C levels. Evolocumab and alirocumab have recently been approved by the Therapeutic Goods Administration in Australia for people with FH. Adverse events are generally similar to placebo, but reported side effects include influenza-like reaction, nasopharyngitis, myalgia and raised creatine kinase levels, and there have been reports of neurocognitive side effects (confusion, perception, memory and attention disturbances). ${ }^{34}$ The cost of these agents is likely to be the major limitation to their clinical use. The indications and use of lipoprotein apheresis and other novel therapies, including lomitapide, a microsomal triglyceride transfer protein inhibitor, and mipomersen (an antisense oligonucleotide that targets apolipoprotein B), have been recently reviewed. ${ }^{35}$
Despite the advances reviewed, the implementation and optimisation of models of care for FH remain a major challenge for preventive medicine. Areas of future research should focus on better approaches for detecting $\mathrm{FH}$ in the young and on enhancing the integration of care between GPs and specialists. The value of genetic testing and imaging of pre-clinical atherosclerosis in stratifying risk and personalising therapy merits particular attention. Further, with families now living in a global community, more efficient methods of communication and data sharing are required. This may be enabled by international Web-based registries. ${ }^{36}$ Care for people with FH needs to be incorporated into health policy and planning in all countries. ${ }^{10}$

\section{Conclusion}

There have been significant advances in the care of individuals with $\mathrm{FH}$ over the past 3 years. An integrated model of care has been proposed for primary care in Australia. Progress has also been made in the treatment of $\mathrm{FH}$ with the emergence of PCSK9 inhibitors capable of allowing more patients already on statins to attain therapeutic LDL-c targets and hence redressing the residual risk of atherosclerotic CVD. Future research is required in the areas of models of care, population science and epidemiology, basic science (including genetics), clinical trials, and patient-centric studies. ${ }^{37}$ Finally, the onus rests on all health care professionals to improve the care of families with $\mathrm{FH}$, in order to save lives, relieve suffering and reduce health care expenditure.

Competing interests: Gerald Watts has received payment from Amgen, Sanofi and Regeneron for lectures, membership of advisory boards and research activities.

Provenance: Not commissioned; externally peer reviewed.

(c) 2016 AMPCo Pty Ltd. Produced with Elsevier B.V. All rights reserved.
1 Vallejo-Vaz AJ, Kondapally Seshasai SR, Cole D, et al. Familial hypercholesterolaemia: a global call to arms. Atherosclerosis 2015; 243: 257-259.

2 Watts GF, Sullivan DR, Poplawski N, et al. Familial hypercholesterolaemia: a model of care for Australasia. Atheroscler Suppl 2011; 12: 221-231.

3 Nordestgaard BG, Chapman MJ, Humphries SE, et al. Familial hypercholesterolaemia is underdiagnosed and undertreated in the general population: guidance for clinicians to prevent coronary heart disease: consensus statement of the European Atherosclerosis Society. Eur Heart J 2013; 34: 3478-3490.

4 Sjouke B, Kusters DM, Kindt I, et al. Homozygous autosomal dominant hypercholesterolaemia in the Netherlands: prevalence, genotype-phenotype relationship, and clinical outcome. Eur Heart J 2015; 36: $560-565$

5 Watts GF, Shaw JE, Pang J, et al. Prevalence and treatment of familial hypercholesterolaemia in Australian communities. Int J Cardiol 2015; 185: 69-71.

6 Cuchel M, Bruckert E, Ginsberg HN, et al. Homozygous familial hypercholesterolaemia: new insights and guidance for clinicians to improve detection and clinical management. A position paper from the Consensus Panel on Familial Hypercholesterolaemia of the European Atherosclerosis Society. Eur Heart J 2014; 35: 2146-2157.

7 Goldberg AC, Hopkins PN, Toth PP, et al. Familial hypercholesterolemia: screening, diagnosis and management of pediatric and adult patients: clinical guidance from the National Lipid Association Expert
Panel on Familial Hypercholesterolemia. J Clin Lipidol 2011; 5: 133-140.

8 Wiegman A, Gidding SS, Watts GF, et al. Familial hypercholesterolaemia in children and adolescents: gaining decades of life by optimizing detection and treatment. Eur Heart J 2015; 36: 2425-2437

9 Harada-Shiba M, Arai H, Oikawa S, et al. Guidelines for the management of familial hypercholesterolemia. J Atheroscler Thromb 2012; 19: 1043-1060.

10 Watts GF, Gidding S, Wierzbicki AS, et al. Integrated guidance on the care of familial hypercholesterolaemia from the International FH Foundation. Int J Cardiol 2014; 171: 309-325

11 Bell DA, Pang J, Burrows S, et al. Effectiveness of genetic cascade screening for familial hypercholesterolaemia using a centrally co-ordinated clinical service: an Australian experience. Atherosclerosis 2015; 293: 93-100.

12 Kusters DM, Avis HJ, de Groot E, et al. Ten-year follow-up after initiation of statin therapy in children with familial hypercholesterolemia. JAMA 2014; 312: 1055-1057.

13 Klancar G, Groselj U, Kovac J, et al. Universal screening for familial hypercholesterolemia in children. J Am Coll Cardiol 2015; 66: 1250-1257.

14 Ademi Z, Watts GF, Pang J, et al. Cascade screening based on genetic testing is cost-effective: evidence for the implementation of models of care for familial hypercholesterolemia. J Clin Lipidol 2014; 8: 390-400.

15 Bell DA, Hooper AJ, Bender R, et al. Opportunistic screening for familial hypercholesterolaemia via a community laboratory. Ann Clin Biochem 2012; 49(Pt 6): 534-537.

16 Bell DA, Bender R, Hooper AJ, et al. Impact of interpretative commenting on lipid profiles in people at high risk of familial hypercholesterolaemia. Clin Chim Acta 2013; 422: 21-25.

17 Bell DA, Hooper AJ, Edwards G, et al. Detecting familial hypercholesterolaemia in the community: Impact of a telephone call from a chemical pathologist to the requesting general practitioner. Atherosclerosis 2014; 234: 469-472

18 Bell DA, Garton-Smith J, Vickery A, et al. Familial hypercholesterolaemia in primary care: knowledge and practices among general practitioners in Western Australia. Heart Lung Circ 2014; 23: 309-313.

19 Kirke AB, Barbour RA, Burrows S, et al. Systematic detection of familial hypercholesterolaemia in primary health care: a community based prospective study of three methods. Heart Lung Circ 2015; 24: $250-256$.

20 Vickery AW, Bell D, Garton-Smith J, et al. Optimising the detection and management of familial hypercholesterolaemia: central role of primary care and its integration with specialist services. Heart Lung Circ 2014; 23: 1158-1164.

21 Troeung L, Arnold-Reed D, Ping-Delfos WCS, et al. A new electronic tool for identifying risk of familial hypercholesterolaemia in general practice. Heart 2016; 102: 855-861.

22 Khera AV, Won H-H, Peloso GM, et al. Diagnostic yield and clinical utility of sequencing familial 
hypercholesterolemia genes in patients with severe hypercholesterolemia. J Am Coll Cardiol 2016; 67: 2578-2589.

23 Hooper AJ, Nguyen LT, Burnett JR, et al. Genetic analysis of familial hypercholesterolaemia in Western Australia. Atherosclerosis 2012; 224: 430-434.

24 Talmud PJ, Shah S, Whittall R, et al. Use of low-density lipoprotein cholesterol gene score to distinguish patients with polygenic and monogenic familial hypercholesterolaemia: a case-control study. Lancet 2013; 381: 1293-1301.

25 Futema M, Plagnol V, Li K, et al. Whole exome sequencing of familial hypercholesterolaemia patients negative for LDLR/APOB/PCSK9 mutations. J Med Genet 2014; 51: 537-544.

26 Norsworthy PJ, Vandrovcova J, Thomas ER, et al. Targeted genetic testing for familial hypercholesterolaemia using next generation sequencing: a population-based study. BMC Med Genet 2014; 15: 70
27 Watts GF, Wierzbicki AS. The hinterland of familial hypercholesterolaemia: what do we not know? Curr Opin Lipidol 2015; 26: 475-483.

28 Chan DC, Pang J, Hooper AJ, et al. Elevated lipoprotein(a), hypertension and renal insufficiency as predictors of coronary artery disease in patients with genetically confirmed heterozygous familial hypercholesterolemia. Int J Cardiol 2015; 201: 633-638.

29 Kamstrup PR, Tybjærg-Hansen A, Nordestgaard BG. Extreme lipoprotein(a) levels and improved cardiovascular risk prediction. J Am Coll Cardiol 2013; 61: 1146-1156.

30 Alonso R, Andres E, Mata N, et al. Lipoprotein(a) levels in familial hypercholesterolemia: an important predictor of cardiovascular disease independent of the type of LDL receptor mutation. J Am Coll Cardiol 2014; 63 1982-1989.

31 Nordestgaard BG, Chapman MJ, Ray K, et al. Lipoprotein(a) as a cardiovascular risk factor: current status. Eur Heart J 2010; 31: 2844-2853.
32 Cannon CP, Blazing MA, Giugliano RP, et al. Ezetimibe added to statin therapy after acute coronary syndromes. N Engl J Med 2015; 372: 2387-2397.

33 Farnier M. PCSK9: from discovery to therapeutic applications. Arch Cardiovasc Dis 2014; 107: 58-66.

34 Navarese EP, Kolodziejczak M, Schulze V, et al. Effects of proprotein convertase subtilisin/kexin type 9 antibodies in adults with hypercholesterolemia: a systematic review and meta-analysis. Ann Intern Med 2015; 163: 40-51.

35 Page MM, Bell DA, Hooper AJ, et al. Lipoprotein apheresis and new therapies for severe familial hypercholesterolemia in adults and children. Best Pract Res Clin Endocrinol Metab 2014; 28: 387-403.

36 Hammond E, Watts GF, Rubinstein Y, et al. Role of international registries in enhancing the care of familial hypercholesterolaemia. Int J Evid Based Healthc 2013; 11: 134-139.

37 Gidding SS, Champagne MA, de Ferranti SD, et al. The agenda for familial hypercholesterolemia: a scientific statement from the American Heart Association. Circulation 2015; 132: 2167-2192. 